\title{
Vertebral artery dominance contributes to basilar artery curvature and peri-vertebrobasilar junctional infarcts
}

\author{
J M Hong, ${ }^{1}$ C-S Chung, ${ }^{2}$ O Y Bang, ${ }^{2}$ S W Yong, ${ }^{1}$ I S Joo, ${ }^{1} \mathrm{~K}$ Huh ${ }^{1}$
}

See Editorial Commentary, p 1057

${ }^{1}$ Department of Neurology, Ajou University Medical Centre, Suwon, South Korea;

${ }^{2}$ Department of Neurology, Samsung Medical Centre, Sungkyunkwan University School of Medicine, Seoul, South Korea

Correspondence to: Dr J M Hong, Department of Neurology, Ajou University

Medical Centre, Ajou University School of Medicine, 5 San, Woncheon-dong, Yongtong-gu, Suwon-si, Kyunggi-do, 442-749, South Korea; dacda@hanmail. net

Received 5 December 2008 Revised 23 February 2009 Accepted 2 April 2009 Published Online First 3 May 2009

\section{(2) UNLOCKAD}

This paper is freely available online under the BMJ Journals unlocked scheme, see http:// jnnp.bmi.com/info/unlocked.dtl

\begin{abstract}
Objectives: The diameters of the vertebral arteries (VAs) are very often unequal. Therefore, this study investigated if unequal VA flow contributes to the development of basilar artery (BA) curvature and if it is a link to the laterality of pontine or cerebellar infarcts occurring around the vertebrobasilar junction.
\end{abstract}

Methods: Radiological factors were analysed (infarct laterality, VA dominance, BA curvature and their directional relationships) in 91 patients with acute unilateral pontine or posterior inferior cerebellar artery (PICA) territory infarcts. The "dominant" VA side was defined as either that the VA was larger in diameter or the VA was connected with the BA in more of a straight line, if both VAs looked similar in diameter on CT angiography. Multiple regression analysis was performed to predict moderate to severe BA curvature.

Results: The dominant VA was more frequent on the left side $(p<0.01)$. Most patients had an opposite directional relationship between the dominant VA and BA curvature $(p<0.01)$. Pontine infarcts were opposite to the side of BA curvature $(p<0.01)$ and PICA infarcts were on the same side as the non-dominant VA side $(p<0.01)$. The difference in VA diameters was the single independent predictor for moderate to severe BA curvature (OR per $1 \mathrm{~mm}, 2.70 ; 95 \% \mathrm{Cl} 1.22$ to 5.98 ).

Conclusions: Unequal VA flow is an important haemodynamic contributor of BA curvature and development of peri-vertebrobasilar junctional infarcts.

Atherosclerosis is a chronic, inflammatory, fibroproliferative systemic disease primarily of large and medium sized conduit arteries. ${ }^{1}$ Atherosclerotic plaques tend to develop in focal regions with complicated flow patterns, or with low or oscillatory wall shear stress (WSS) as in regions such as bifurcations, bends and junctions. ${ }^{2-4}$ Mechanical forces acting on the arterial wall, including the WSS, are thought to be local factors influencing the development of atherosclerosis and regulating vessel calibre and morphology (ie, vascular remodelling). ${ }^{5}$

The basilar artery (BA), which supplies the brainstem and posterior part of the human brain, arises from the junction of the two vertebral arteries (VAs). Unlike most systemic arteries, which have a tree-like branching pattern, the BA is the only large artery in which two arterial flows merge. Few studies have examined the flow dynamics at the vertebrobasilar junction. ${ }^{36}$ In addition, the diameters of the VAs are of equal size in only $6-26 \%$ of patients in angiographic or postmortem studies, and the left VA is often larger than the right VA. ${ }^{7}$ Therefore, we postulated that the unequally mechanical forces resulting from asymmetric VA flow might influence the morphological deformation in the vertebrobasilar arterial system (lateral displacement or elongation of the BA, hypoplasia of the VA), and by extension, such deformations might asymmetrically induce the development of infarcts in the areas before or after the vertebrobasilar junction.

To test this hypothesis, we investigated the demographics, specific locations of unilateral infarcts around vertebrobasilar junction, radiological findings and haemodynamic findings. We also analysed the potential predictors of the moderate to severe BA curvature.

\section{PATIENTS AND METHODS}

\section{Patients}

From January 2005 to June 2007, we retrospectively identified all consecutive acute ischaemic stroke patients admitted to two tertiary referral medical centres. We included patients if they had a final diagnosis of acute ischaemic stroke with diffusion weighted imaging (DWI) confirmation of lesions involving the posterior inferior cerebellar artery (PICA) territory unilaterally or pons unilaterally. A detailed history of vascular risk factors was obtained from each patient. To identify the potential mechanism of cerebral infarcts, a set of diagnostic tests was performed that included CT angiography, routine blood tests and a cardiology workup (ECG and echocardiogram). MRI was performed in all patients with a $1.5 \mathrm{~T}$ scanner (GE Medical, USA) and scanning with conventional T2 weighted MRI and DWI was conducted in the axial plane using $7 \mathrm{~mm}$ thick sections.

Based on the results of the vascular and cardiology studies, we divided the patients' stroke mechanisms into six groups: cardioembolism, large artery atherosclerosis, small artery disease, other determined aetiologies (eg, vasculitis or dissection), undetermined aetiology and coexisting aetiologies ( $>1$ cause). We excluded patients: (1) if their mechanisms fell into the cardioembolism, other determined cause or coexisting aetiology group, other determined cause or coexisting aetiology; (2) if the VA or BA was not seen on angiographic study; or (3) if the pontine lesion coexisted with the PICA territorial lesion.

To compare the vascular anatomical status (the morphology of the BA and VA) of patients with posterior circulation ischaemic stroke with those of age matched populations, we evaluated brain CT angiography of those patients aged over 40 years 
Table 1 Clinical and radiological characteristics of controls $(n=83)$ and patients $(n=91)$ with unilateral pontine or PICA infarcts

\begin{tabular}{|c|c|c|c|}
\hline & $\begin{array}{l}\text { Controls } \\
(\mathrm{n}=83)\end{array}$ & $\begin{array}{l}\text { Patients } \\
(\mathrm{n}=91)\end{array}$ & p Value \\
\hline \multicolumn{4}{|l|}{ General demographic data } \\
\hline Age (year) (mean (SD)) & $62.5(11.7)$ & $63.6(11.6)$ & 0.526 \\
\hline Sex $(\%$ men $)$ & $49(59.0)$ & $55(60.0)$ & 0.850 \\
\hline \multicolumn{4}{|l|}{ Risk factors (n (\%)) } \\
\hline Hypertension & & $65(71.4)$ & \\
\hline Diabetes mellitus & & $35(38.5)$ & \\
\hline Smoking & & $30(33.0)$ & \\
\hline Previous stroke & & $18(19.8)$ & \\
\hline Hyperlipidaemia & & $22(24.2)$ & \\
\hline \multicolumn{4}{|l|}{ Direction of vascular deformations } \\
\hline Direction of dominant flow VA (n) & & & 0.803 \\
\hline $\mathrm{R}$ side & 27 & 28 & \\
\hline L side & 56 & 63 & \\
\hline Direction of BA curvature (n) & & & 0.007 \\
\hline $\mathrm{R}$ side & 45 & 68 & \\
\hline L side & 29 & 21 & \\
\hline Visually straight BA & 9 & 2 & \\
\hline Degree of BA curvature $(n)$ & & & $<0.001$ \\
\hline Grade 0 & 9 & 2 & \\
\hline Grade 1 & 65 & 41 & \\
\hline Grade 2 & 9 & 35 & \\
\hline Grade 3 & 0 & 13 & \\
\hline Degree of BA height (n) & & & $<0.001$ \\
\hline Grade 1 & 82 & 73 & \\
\hline Grade 2 & 1 & 17 & \\
\hline Grade 3 & 0 & 1 & \\
\hline \multicolumn{4}{|l|}{ Diameter of arteries $(\mathrm{mm})$} \\
\hline BA (mean (SD)) & $3.17(0.70)$ & $3.20(0.56)$ & 0.759 \\
\hline R VA (mean (SD)) & $2.35(0.82)$ & $1.95(0.68)$ & 0.001 \\
\hline L VA (mean (SD)) & $2.48(0.72)$ & $2.48(0.74)$ & 0.998 \\
\hline
\end{tabular}

BA, basilar artery; L, left; PICA, posterior inferior cerebellar artery; R, right; VA, vertebral artery.

with no history of stroke who visited the outpatient clinic of the Department of Neurology, Ajou University Hospital from January 2007 to June 2007. The study was approved by the Institutional Review Board at Ajou University Hospital.

\section{Imaging analysis}

Topographical determination of acute unilateral cerebellar infarction in the PICA territory was performed using visual correlation between Amarenco's templates and the locations of high signal intensities from DWI that were more than $2 \mathrm{~cm}$ in diameter. ${ }^{8}$ Two of the authors (JMH and CSC) came to a topographical consensus. We defined acute unilateral pontine infarction as DWI lesions involving the pons unilaterally. To evaluate the frequency of affected sites in the cerebellum and pons, we made contour maps using MRIcro software (C Rorden, www.mricro.com).

The diameter of each vessel was calculated as the average of the measurements made at three consecutive points, $3 \mathrm{~mm}$ apart, starting from the vertebrobasilar junction (both VAs and the BA). The "dominant" VA was defined as (1) having the larger diameter within a strict criterion for diameter (ie, a side to side diameter difference $\geqslant 0.3 \mathrm{~mm})^{7}$ or as (2) the VA connected to the BA in a more straight fashion if both VAs were visually similar to a criterion of angle on CT angiography.

The direction of BA curvature was designated as "right (R)" or "left (L) side" according to a course of BA navigation at the vertebrobasilar junction. The degree of $\mathrm{BA}$ curvature was evaluated using a previously suggested CT based method, ${ }^{9}$ based on the lateral-most position of the BA throughout its course ( 0 , midline; 1 ( $R$ or $L)$, medial to lateral margin of the clivus or dorsum sellae; 2 ( $R$ or $L$ ), lateral to the lateral margin of the clivus or dorsum sellae; and 3 , in the cerebellopontine angle cistern). Moderate to severe BA curvature was defined as $\geqslant 2$ of the above criteria. The height of the bifurcation of the BA was scored as:1, within the suprasellar cistern; 2 , at the level of the third ventricle floor; and 3 , indenting and elevating the floor of the third ventricle.

\section{Transcranial Doppler and wall shear stress analysis}

Transcranial Doppler was performed with a $2 \mathrm{MHz}$ pulsed Doppler instrument (EME TC-8080, USA) by an experienced technician within 7 days of admission. Patients were asked to relax and breathe normally while in a comfortable supine position in a quiet room. BA flow was identified by the signal directed away from the probe through the suboccipital window

(A)
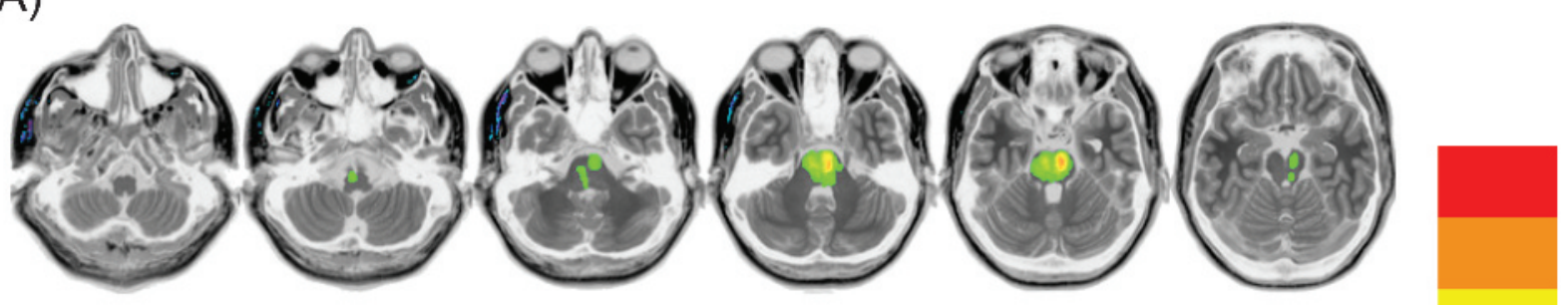

$>40 \%$

$30-40 \%$

(B)

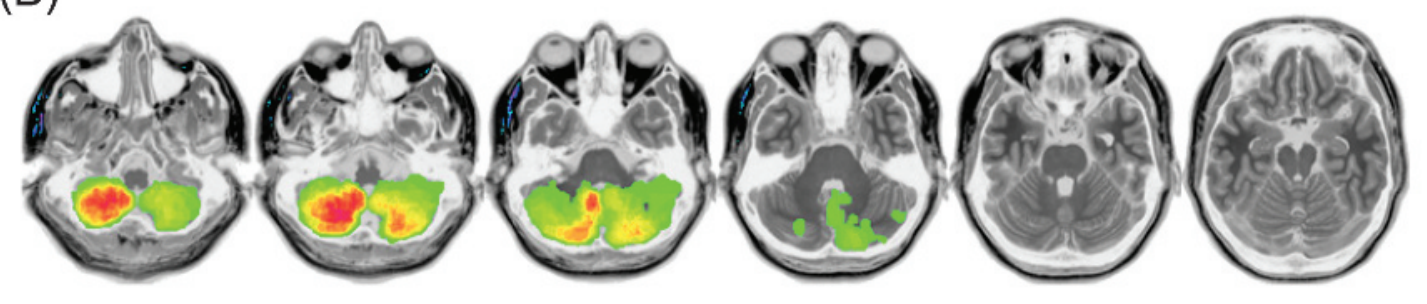

$20-30 \%$

$10-20 \%$

$<10 \%$

Figure 1 Overlapping contour maps of peri-vertebrobasilar junctional infarcts. Paramedian region of the left mid-pons was the most affected site for post-vertebrobasilar junctional infarcts (ie, pontine infarcts) whereas the right inferior medial region in the cerebellum was the most affected site for the ischaemic infarcts around the pre-vertebrobasilar junction (ie, posterior inferior cerebellar artery infarcts). 
and was evaluated at a depth between 80 and $100 \mathrm{~mm}$ through a suboccipital window. After the probe was shifted a few centimetres inferolaterally, it detected VA flow signals directed away from the probe at depths of $40-75 \mathrm{~mm}$.

Based on the assumption of laminar blood flow in the setting of a Poiseuillian parabolic model, we calculated the mean wall shear rate (WSR): WSR $=4 \times$ mean blood flow velocity/ internal diameter. ${ }^{10}$ WSR was also calculated for mean shear rate. Whole blood viscosity $\left(\eta_{w}\right)$ was estimated from general plasma viscosity at $37^{\circ} \mathrm{C}\left(\eta_{0}=1.5 \mathrm{cP}\right)$, and haematocrit $(\mathrm{Ht})$ of the patients was determined using Einstein's equation $\left[\eta_{w}=\eta_{0}\right.$ $(1+2.5 \mathrm{Ht})]$. Mean wall shear stresses were calculated using the formula: WSS $=\eta_{w} \times$ WSR $^{11}{ }^{12}$

\section{Statistical analysis}

Differences between groups (patients vs controls, right vs left, dominant vs non-dominant VA side or each tertile according to the diameter difference of the VAs) were analysed using the Student's $t$ test, $\chi^{2}$ test, paired t test or analysis of variance, as appropriate for continuous and categorical variables. We performed a multiple regression analysis to determine which variables were independent predictors of moderate to severe BA curvature. All potential predictors were entered into a univariate logistic regression model, including demographic variables (ie, age, sex and risk factors for stroke) and radiological/haemodynamic variables (ie, $\mathrm{R}$ or $\mathrm{L}$ VAs diameter, BA diameter, diameter difference of VAs) and WSS (ie, dominant VA, non-dominant $\mathrm{VA}$ and BA). Potential factors that were not significant $(p>0.2)$ in the univariate analysis were sequentially deleted from the full multivariable model. Results are given as odds ratio (OR), as estimates of the relative risk with $95 \%$ confidence interval (CI). Statistical significance was considered at $p<0.05$.

\section{RESULTS}

\section{General demographics and radiological and haemodynamic findings}

Of 133 consecutive patients with an acute unilateral pontine or PICA infarction, 91 were eligible for this study. Forty-one patients were excluded for cardioembolism $(n=26)$, invisible
VA or BA $(n=11)$, VA dissection $(n=3)$, coexistence of pontine and PICA infarcts $(n=1)$ and BA dissection $(n=1)$. Mean age of the patients was 63.6 (11.6) years and 55 (60.4\%) were men.

Table 1 shows the comparative results for age, sex and radiological variables between 83 controls and 91 patients. There were no significant differences in age or sex, direction of dominant flow VA (predominantly left-sided) or diameter of respective arteries (right and left $\mathrm{VA}$, and $\mathrm{BA}$ ) between cases and control; however, there were significant differences in the degree of BA dolichosis and BA height between the two groups. Mean viscosity was 3.06 (0.17) Cp (centipoise, range 2.57$3.45 \mathrm{Cp}$ ) in patients. Mean WSS of the VAs was significantly higher on the right side than on the left (10.4 (6.8) vs 8.0 (5.2) dyn $\left./ \mathrm{cm}^{2} ; \mathrm{p}=0.009\right)$, especially on the non-dominant flow VA side (11.3 (7.4) vs $\left.7.1(3.4) \mathrm{dyn} / \mathrm{cm}^{2} ; \mathrm{p}<0.001\right)$. On the contralateral side, no difference was observed in mean WSS according to BA depth between 80 and $90 \mathrm{~mm}$ (7.6 (4.4) vs 7.6 (4.7) $\left.\mathrm{dyn} / \mathrm{cm}^{2} ; \mathrm{p}=0.965\right)$.

\section{Directional relationships of the radiological findings}

The contour maps of the affected sites in the pons and PICA cerebellum are depicted in fig 1 . We observed 47 pontine infarcts (R 20 vs L 27) and 44 PICA infarcts (R 26 vs L 18). For pontine infarcts, the paramedian region of the left mid-pons was the most affected site whereas the right inferior medial region in the cerebellum was the most affected site for the PICA infarcts.

The dominant VA side was determined by the diameter criteria in $84(92.3 \%)$ patients, and the rest of the patients by criteria of angle. The dominant VA was more frequent on the left VA side $(69.2 \%$; $p 00.001)$. BA curvature was mainly directed to the right side and the most frequent morphological change in the BA was a C-shaped deformation $(n=65)$, followed by S-shaped $(n=17)$, J-shaped $(n=7)$ and no deformation or straight $(n=2)$. Forty-eight patients had moderate to severe curvature of the $\mathrm{BA}$ and 18 had elongation of the BA. Eighty-six patients had lateral displacement of the BA ( $\geqslant$ grade 1 ) and a directional relationship was detected between the dominant VA side and the BA curvature in the opposite direction in $76(83.5 \%)$ patients $(76 / 91 ; \mathrm{p}<0.001)$.
Figure 2 Directional relationships according to BA angulation, VA dominancy and peri-vertebral junctional infarcts (ie, pontine and PICA infarcts). BA, basilar artery, PICA, posterior inferior cerebellar artery; VA, vertebral artery.

\section{-BA angulation}

- $B A$ curvature of grade $\geq 1$; ( $n=89,97.8 \%)$

- BA curvature of grade $\geq 2$; $(n=48,52.7 \%)$

- BA elongation of grade $\geq 2$; $(n=18,19.8 \%)$

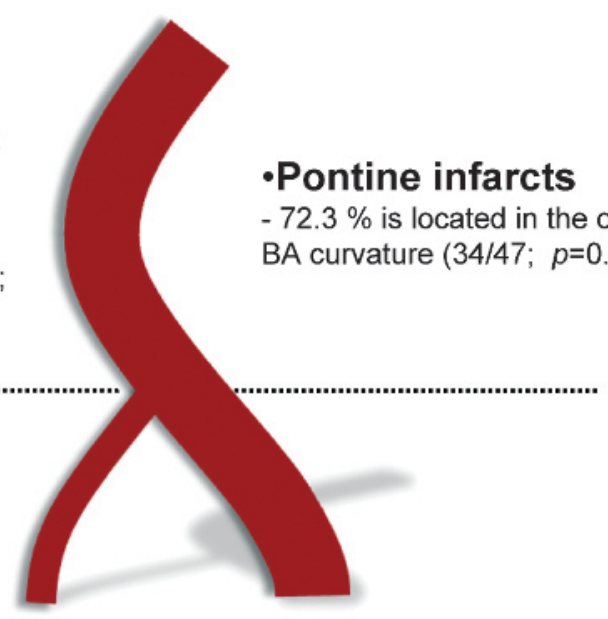

-Dominant VA

$-69.2 \%$ is located in the left VA side $(63 / 91$; $p<0.001$ )

- Patients having dominant VA is opposite to BA curvature are $83.5 \%(76 / 91 ; p<0.001)$ 
Figure 2 shows that pontine infarcts opposite to the side of BA curvature occupied $72.3 \%(34 / 47 ; p=0.002)$ while PICA infarcts on the same side of the non-dominant VA occupied $72.7 \%(32 / 44 ; p=0.005)$.

\section{Relationship between diameter difference of VAs and BA dolichosis}

Patient demographics according to the diameter difference of the VAs are shown in table 2. The number of patients with hypertension and moderate to severe BA curvature was more and more prevalent towards a higher tertile of VA diameter differences. The mean diameter of the right VA gradually decreased in the higher tertile group. Mean WSSs on the right and particularly on the non-dominant VA increased in the higher tertile group of VA diameter differences.

Hypertension, older age and the diameter difference of the VAs were associated with moderate to severe BA curvature in univariate analysis. After adjusting for all potential variables, only the diameter difference of the VAs remained as an independent predictor of moderate to severe BA curvature in the full multivariate model (OR per $1 \mathrm{~mm}$ increase $2.70,95 \% \mathrm{CI}$ 1.22 to $5.98 ; p=0.015)$. Other haemodynamic variables, such as viscosity, other vessel diameters, WSR and WSS of vessels, were not significantly associated with moderate to severe BA curvature.

\section{DISCUSSION}

We found that pontine infarcts more frequently occurred opposite to the side of BA curvature and PICA infarcts were more frequent on the non-dominant VA side, and that the difference in the diameters of the right and left VA was the only independent predictor for moderate to severe BA curvature. Hence the present study suggested that the asymmetrical flow pattern of VAs around the vertebrobilar junction might be an important mechanical force in the origin of BA curvature as well as a causative factor of peri-vertebrobasilar junctional infarcts (ie, pontine and PICA infarcts).

In this study, VA diameter was significantly larger on the left than on the right side, which is consistent with numerous previous reports. ${ }^{7314}$ Traditionally, most physicians have regarded an asymmetric VA as a congenital variant or a clinically meaningless finding, unless vertebrobasilar insufficiency occurs. ${ }^{15} 16$ However, recent studies have regarded VA hypoplasia as a risk factor for posterior circulation stroke, and that its directional association with a stroke had an ipsilateral tendency to VA hypoplasia..$^{17-19}$ Interestingly, we also found that a specific stroke (ie, pontine infarction) had a tendency to the opposite side to the lateral displacement of the BA. In addition, the curvature of the BA was directionally opposite to the dominant VA.

Vertebrobasilar dolichoectasia is anatomically well known and appears to be postulated as a risk factor for posterior circulation stroke. ${ }^{20-22}$ Currently, a study reported on the directional relationship between vertebrobasilar dolichoectasia and the specific locations of infarcts in the vertebrobasilar system-that is, $58 \%$ of patients $(11 / 19)$ had pontine or cerebellar lesions (superior and anterior cerebellar lesions) contralateral to the lateral displacement of the BA versus ipsilateral in $26 \%$ of patients $(5 / 19) .{ }^{20} \mathrm{~A}$ recent study on

Table 2 Clinical and haemodynamic characteristics in 91 patients stratified by tertiles according to diameter difference of the VAs

\begin{tabular}{|c|c|c|c|c|}
\hline & \multicolumn{3}{|c|}{ Diameter difference of VAs (mm) } & \multirow[b]{2}{*}{ p Value } \\
\hline & $\begin{array}{l}\text { Low } 1 / 3 \\
\text { (range } 0.04 \sim 0.70 \text { ) }\end{array}$ & $\begin{array}{l}\text { Middle 1/3 } \\
\text { (range } 0.71 \sim 1.17 \text { ) }\end{array}$ & $\begin{array}{l}\text { High 1/3 } \\
\text { (range 1.19 2.67) }\end{array}$ & \\
\hline No of cases & 30 & 30 & 31 & \\
\hline Age (year) (mean (SD)) & $62.7(13.0)$ & $62.5(11.0)$ & $66.2(10.4)$ & 0.390 \\
\hline \multicolumn{5}{|l|}{ Risk factors (n (\%)) } \\
\hline Hypertension & $17(56.7)$ & $21(70.0)$ & $27(87.1)$ & $0.031^{*}$ \\
\hline Diabetes mellitus & $11(36.7)$ & $14(46.7)$ & $10(32.3)$ & 0.497 \\
\hline Smoking & $10(33.3)$ & $8(26.7)$ & $12(40.0)$ & 0.547 \\
\hline Previous stroke & $8(26.7)$ & $5(16.7)$ & $5(16.1)$ & 0.512 \\
\hline \multicolumn{5}{|l|}{ Initial laboratory findings (mean (SD)) } \\
\hline Haemoglobin (g/dl) & $14.3(1.6)$ & $13.6(1.5)$ & $14.4(1.8)$ & 0.166 \\
\hline Glucose (mg/dl) & $137.7(48.0)$ & $171.0(74.1)$ & $151.7(67.1)$ & 0.138 \\
\hline Total cholesterol (mg/dl) & $176.0(36.4)$ & $171.8(45.8)$ & $180.4(41.7)$ & 0.984 \\
\hline$C$ reactive protein $(\mathrm{mg} / \mathrm{dl})$ & $0.36(0.51)$ & $0.80(2.18)$ & $0.69(1.49)$ & 0.512 \\
\hline Fibrinogen (mg/dl) & $314.3(72.6)$ & $357.4(88.3)$ & $324.9(74.2)$ & 0.112 \\
\hline \multicolumn{5}{|l|}{ Radiological findings } \\
\hline M-to-S BA curvature (n (\%)) & $10(33.3)$ & $15(50.0)$ & $23(74.2)$ & $0.006^{*}$ \\
\hline M-to-S BA elongation (n (\%)) & $4(13.3)$ & $9(30.0)$ & $5(16.1)$ & 0.221 \\
\hline Diameter of BA (mm) (mean (SD)) & $3.15(0.58)$ & $3.16(0.56)$ & $3.27(0.55)$ & 0.660 \\
\hline Diameter of R VA (mm) (mean (SD)) & $2.29(0.38)$ & $1.98(0.56)$ & $1.58(0.86)$ & $0.001 *$ \\
\hline Diameter of L VA (mm) (mean (SD)) & $2.35(0.60)$ & $2.52(0.57)$ & $2.53(0.97)$ & 0.589 \\
\hline \multicolumn{5}{|l|}{ WSS (dyn/ $\left.\mathrm{cm}^{2}\right)$ (mean (SD)) } \\
\hline WSS in R VA & $7.1(2.0)$ & $11.2(7.7)$ & $14.0(8.2)$ & $0.004^{*}$ \\
\hline WSS in L VA & $6.9(2.8)$ & $9.1(8.2)$ & $8.5(4.4)$ & 0.407 \\
\hline WSS in BA at $80 \mathrm{~mm}$ & $6.6(4.0)$ & $8.3(5.5)$ & $8.2(4.0)$ & 0.429 \\
\hline WSS in BA at $90 \mathrm{~mm}$ & $6.4(5.1)$ & $8.5(4.8)$ & $8.4(3.8)$ & 0.308 \\
\hline WSS in dominant VA & $6.6(2.4)$ & $8.1(5.3)$ & $6.9(2.6)$ & 0.449 \\
\hline WSS in non-dominant VA & $7.4(2.5)$ & $12.3(9.5)$ & $15.6(7.5)$ & $0.001^{*}$ \\
\hline
\end{tabular}

BA, basilar artery; L, left; M-to-S, moderate to severe; R, right; VA, vertebral artery; WSS, wall shear stress. ${ }^{*} \mathrm{p}<0.05$. 
Figure 3 Schematic illustrations of the pathophysiological process of perivertebral junctional infarcts: possible changes in vetebrobasilar vessels under the condition of an unequal VA flow. BA, basilar artery, VA, vertebral artery.

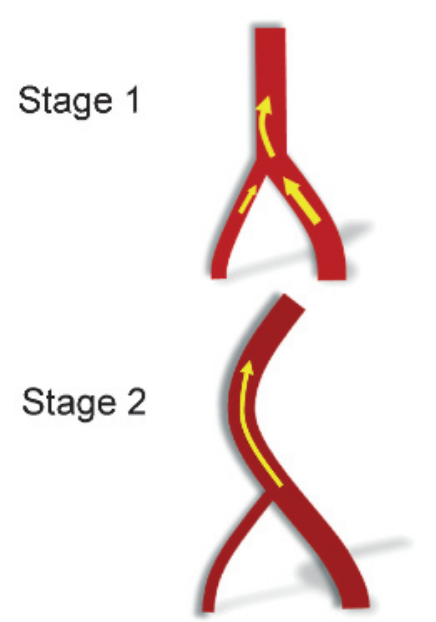

- Congenitally asymmetric flow of VAs with straight $\mathrm{BA}$

\section{- Curving or elongation of BA caused by asymmetric wall tension}

- Non-dominant VA can lead to be narrower due to direct decrease of flow or easy collapse by Bernoulli's effect under the condition of decreased vascular remodeling capacity

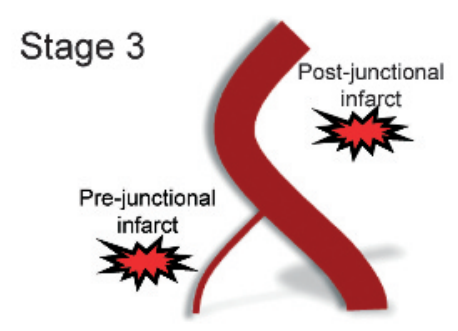

- Eventually, pre-junctional infarctions in nondominant VA side, and post-junctional infarctions frequently occur in opposite site to the BA curvature (eq. more thrombogenic at the inner bending portion of BA or traction of pontine perforators) consecutive series of cerebellar infarcts showed that cerebellar infarcts at the post-vertebrobasilar junction (anterior inferior cerebellar artery and superior cerebellar artery territory) were more common on the left side. This study also indicated that there was a right predilection at the pre-vertebrobasilar junction (PICA territory) and a left predilection at the post-vertebrobasilar junction (superior cerebellar artery territory) even in patients with combined involvements of cerebellar arterial territories. ${ }^{23}$ For these reasons, our findings may provide more evidence that the specific location of infarcts is closely connected to BA curvature or VA hypoplasia.

Such directional relationships can be explained by several haemodynamic mechanisms. Firstly, the inner wall of the BA curvature may be more thrombogenic because of a low WSS, ${ }^{4} 4$ and traction of the pontine perforators caused by BA curvature may lead to infarction. ${ }^{20}$ Secondly, a hypoplastic VA can cause ipsilateral PICA infarction by directly decreasing blood flow in the smaller intracranial VA. ${ }^{24}$ This can occur because of the easy collapsibility of a narrowed vessel as a result of Bernoulli's effect under the decreased VA remodelling capacity. ${ }^{25}$ Our theory is outlined in fig 3 . The vector of BA flow merging from unequal VAs makes the BA flow curve to the side of the weaker VA, and the chronic processes caused by asymmetric VA flow can induce greater curving of the BA wall. Subsequently, such deformation of the BA can cause atherogenesis, leading to ischaemic stroke in the vertebrobasilar system. A hypoplastic VA can also result in the ipsilateral occlusion of this vessel due to a direct decrease in blood flow and easy collapse of the vessel caused by the smaller intracranial VA calibre.

The diameter difference of the VAs was the only independent predictor for the moderate to severe BA dolichosis, even after adjusting for confounding variables (demographics, radiological variables and haemodynamic variables). In general, the flow rate is proportional to the fourth power of the vessel radius, according to Poiseuille's law. ${ }^{24}$ Therefore, the radius of the vessel is the most essential determinant of blood flow, even in the vertebrobasilar system. Pressure, blood flow or both factors are widely recognised as potential stimuli for morphological change or the functional adaptation of vessels. ${ }^{26}$ Numerous studies have examined flow dependent remodelling, ${ }^{26-29}$ and if such haemodynamic forces are altered chronically, a subsequent morphological or structural adaptation of the artery can occur to minimise the effect of the altered haemodynamic forces on the vascular wall, including changes in calibre and wall thickness.

Our study has several limitations. We investigated the values of WSSs using pulsed Doppler sonography. Although it is a simple, easy and non-invasive way for the assessment of WSS, it can be technically limited as it is unable to visualise the vessel and also has an angle correction problem. Additionally, it is necessary to assume a linear velocity distribution of the flow with the central peak velocity to measure the wall shear rate. In future studies, accurate and timely measurement of WSS with good spatial resolution should be used. Further research involving vessel remodelling mediators, such as matrix metalloproteinases and nitric oxide, suggesting a role for vascular adaptation, is needed to assess the regulatory aspects of the vessel in response to the flow related mechanical forces. ${ }^{27}$

In conclusion, this study suggests that unequal blood flow from bilateral VAs is a significant haemodynamic contributor to BA curvature. Moreover, it is a potential determinant for the development of acute infarcts around the vertebrobasilar junction.

Competing interests: None.

Ethics approval: This study was approved by the Institutional Review Board at Ajou University Hospital.

Provenance and peer review: Not commissioned; externally peer reviewed.

\section{REFERENCES}

1. Hansson GK. Inflammation, atherosclerosis, and coronary artery disease. N Engl J Med 2005;352:1685-95.

2. Resnick N, Yahav H, Shay-Salit A, et al. Fluid shear stress and the vascular endothelium: For better and for worse. Prog Biophys Mol Biol 2003;81:177-99. 
3. Ravensbergen J, Ravensbergen JW, Krijger JK, et al. Localizing role of hemodynamics in atherosclerosis in several human vertebrobasilar junction geometries. Arterioscler Thromb Vasc Biol 1998;18:708-16.

4. Cunningham KS, Gotlieb Al. The role of shear stress in the pathogenesis of atherosclerosis. Lab Invest 2005;85:9-23.

5. Malek AM, Alper SL, Izumo S. Hemodynamic shear stress and its role in atherosclerosis. JAMA 1999;282:2035-42.

6. Ravensbergen J, Krijger JK, Hillen B, et al. The influence of the angle of confluence on the flow in a vertebro-basilar junction model. J Biomech 1996;29:281-99.

7. Jeng JS, Yip PK. Evaluation of vertebral artery hypoplasia and asymmetry by colorcoded duplex ultrasonography. Ultrasound Med Biol 2004;30:605-9.

8. Amarenco $\mathbf{P}$, Kase CS, Rosengart A, et al. Very small (border zone) cerebellar infarcts. Distribution, causes, mechanisms and clinical features. Brain 1993;116:161-86.

9. Smoker WR, Price MJ, Keyes WD, et al. High-resolution computed tomography of the basilar artery: 1. Normal size and position. AJNR Am J Neuroradiol 1986; 7:55-60.

10. Kornet L, Hoeks AP, Lambregts J, et al. In the femoral artery bifurcation, differences in mean wall shear stress within subjects are associated with different intima-media thicknesses. Arterioscler Thromb Vasc Biol 1999;19:2933-9.

11. Simon AC, Levenson J. Abnormal wall shear conditions in the brachial artery of hypertensive patients. J Hypertens 1990;8:109-14.

12. Jiang $\mathbf{Y}$, Kohara $\mathrm{K}$, Hiwada K. Association between risk factors for atherosclerosis and mechanical forces in carotid artery. Stroke 2000;31:2319-24.

13. Perren F, Poglia D, Landis T, et al. Vertebral artery hypoplasia: A predisposing factor for posterior circulation stroke? Neurology 2007;68:65-7.

14. Min JH, Lee YS. Transcranial doppler ultrasonographic evaluation of vertebral artery hypoplasia and aplasia. J Neurol Sci 2007;260:183-7.

15. Cloud GC, Markus HS. Diagnosis and management of vertebral artery stenosis. OJM 2003;96:27-54.
16. Trattnig S, Hubsch P, Schuster $\mathrm{H}$, et al. Color-coded doppler imaging of normal vertebral arteries. Stroke 1990;21:1222-5.

17. Chuang YM, Huang YC, Hu HH, et al. Toward a further elucidation: Role of vertebral artery hypoplasia in acute ischemic stroke. Eur Neurol 2006;55:193-7.

18. Park JH, Kim JM, Roh JK. Hypoplastic vertebral artery: Frequency and associations with ischaemic stroke territory. J Neurol Neurosurg Psychiatry 2007;78:954-8.

19. Caplan LR. Arterial occlusions: Does size matter? J Neurol Neurosurg Psychiatry 2007;78:916

20. Passero S, Filosomi G. Posterior circulation infarcts in patients with vertebrobasilar dolichoectasia. Stroke 1998;29:653-9.

21. Ubogu EE, Zaidat 00 . Vertebrobasilar dolichoectasia diagnosed by magnetic resonance angiography and risk of stroke and death: A cohort study. J Neurol Neurosurg Psychiatry 2004;75:22-6.

22. Passero SG, Rossi S. Natural history of vertebrobasilar dolichoectasia. Neurology 2008;70:66-72.

23. Hong JM, Bang OY, Chung CS, et al. Frequency and clinical significance of acute bilateral cerebellar infarcts. Cerebrovasc Dis 2008;26:541-8.

24. Hademenos GJ, Massoud TF. Biophysical mechanisms of stroke. Stroke 1997;28:2067-77

25. Binns RL, Ku DN. Effect of stenosis on wall motion. A possible mechanism of stroke and transient ischemic attack. Arteriosclerosis 1989:9:842-7.

26. Koller A. Flow-dependent remodeling of small arteries: The stimuli and the sensors are (still) in question. Circ Res 2006:99:6-9.

27. Tronc $\mathbf{F}$, Mallat Z, Lehoux $\mathrm{S}$, et al. Role of matrix metalloproteinases in blood flowinduced arterial enlargement: Interaction with no. Arterioscler Thromb Vasc Biol 2000:20:E120-6.

28. Korshunov VA, Berk BC. Flow-induced vascular remodeling in the mouse: A model for carotid intima-media thickening. Arterioscler Thromb Vasc Biol 2003;23:2185-91.

29. Langille BL, O'Donnell F. Reductions in arterial diameter produced by chronic decreases in blood flow are endothelium-dependent. Science 1986;231:405-7.

\section{Drug and Therapeutics Bulletin (DTB)}

\section{Your key source of unbiased, independent advice}

For over 45 years DTB has been an independent, indispensable part of evidence-based clinical practice. DTB offers healthcare professionals detailed assessment of, and practical advice on, individual medicines and other treatments, groups of treatment and the overall management of disease.

DTB is now also available online at http://dtb.bmj.com:

- browse or search all DTB content from the latest issue back to 1994

- email alerting, sophisticated searching, RSS feeds and full text links from cited references

- interactive services such as My Folders for quick access to articles that you have viewed previously and My Searches to save and re-use useful searches

- comment online on any DTB article

To subscribe, or for further information, please visit http://dtb.bmj.com 